\title{
The delta high-density lipoprotein cholesterol ratio: a novel parameter for gram-negative sepsis
}

\author{
Guoying Zou ${ }^{1,2^{*}}$, Junyu He ${ }^{1,2}$, Biqiong Ren ${ }^{1,2}$, Fei Xu $u^{1,2}$, Guofeng $X u^{1,2}$ and Wenling Zhang ${ }^{3}$
}

*Correspondence:
zouwanshan75@163.com
'Department of Clinical
Laboratory, Brain Hospital
of Hunan Province,
Furong Middle Road 427,
Changsha 410007, Hunan,
People's Republic of China
Full list of author information
is available at the end of the
article

${ }^{*}$ Correspondence: zouwanshan75@163.com Laboratory, Brain Hospita of Hunan Province, People's Republic of China article

\begin{abstract}
Objective: To study changes in blood lipid metabolism in sepsis patients, especially high-density lipoprotein cholesterol (HDL-C) changes in the diagnosis of sepsis and the type of bacteria involved.
\end{abstract}

Methods: Two-hundred-twenty cases of patients with febrile infections were divided into local infection, systemic inflammatory response syndrome or sepsis (sepsis) group. For controls, 81 cases of patients with a healthy check-up were used. Lipid levels and inflammatory state were supervised, and a comparative analysis of patients admitted to the hospital after 1, 5, 10 days was performed.

Results: In patients with sepsis, total cholesterol, HDL-C, and apolipoprotein A 1 (apoA 1) were significantly decreased in this group. Particularly HDL-C was decreased 1 day after admission. Compared with the patients with gram-positive sepsis, HDL-C and apoA1 were significantly reduced in the patients with gram-negative sepsis at admission. The 24-h change ratio of $\mathrm{HDL}-\mathrm{C}$ was different between the gram-negative and gram-positive sepsis patients with a $70.5 \%$ specificity and $76.5 \%$ sensitivity. The area under the curve was 0.744 , and the critical value was $-21.1 \%$.

Conclusions: The sepsis patients had lower HDL-C than the other groups. The 24-h change ratio of $\mathrm{HDL}-\mathrm{C}$ can be used as a sepsis diagnosis maker and to distinguish between the bacteria involved in sepsis.

Keywords: Gram-negative sepsis, High-density lipoprotein, Bacterial infections

\section{Background}

A bacterial infection is an acute infection of growing pathogenic bacteria or conditional pathogenic bacteria that invade the blood circulation and produce toxins and other metabolites. Pathogenic bacteria can include gram-negative bacteria (gram ${ }^{-}$) and grampositive bacteria $\left(\mathrm{gram}^{+}\right)$. Bacterial infections can easily develop into sepsis in older children with poor immune systems if the infections are left untreated or have complications. Complications are regarded as the main reason for high morbidity and mortality. As the abuse of antibiotics and the difference of the immune system, the traditional diagnostic indicators for bacterial infection or sepsis are difficult to meet the clinical requirements in the matter of sensitivity and specificity, such as temperature, white blood count 
and erythrocyte sedimentation rate. Therefore, better biological indicators are needed for early diagnosis and prognosis.

Microbial cultures are the "gold standard" for diagnosing a bacterial infection. However, cultures can only yield results $48-72 \mathrm{~h}$ after receiving samples. Currently, even the results from a Gram stain require 12-24 h or longer. Although PCR can be a fast and reliable method for identifying bacteria for quality assurance (Hospodsky et al. 2010), its use is restricted in certain areas. Despite the availability of acute phase proteins, such as C-reactive protein (CRP), procalcitonin (PCT), and tumor necrosis factor, as sepsis markers, there is no ideal marker that can reliably differentiate between infected and non-infected patients. Lipoproteins and lipids, which have direct immunomodulatory properties, bind and neutralize toxic bacterial substances and could be new candidates as infection markers (Feingold et al. 1995). High-density lipoprotein (HDL), a lipoprotein that participates in the reverse transportation of cholesterol and selectively inhibits type I interferon gene responses, plays a role in regulating innate immune functions during bacterial infections (Suzuki et al. 2010). Apolipoprotein A 1 (apoA 1) is abundant in HDL and can combine a hydrophilic lipotropic alpha helix with lipid A endotoxin and can limit endotoxin activity, thus improving its bacterially toxic effects (Thaveeratitham et al. 2007).

The present study was designed to investigate changes in HDL level values or ratios in patients with sepsis. HDL can be checked by routine clinical testing (receiving results during $1 \mathrm{~h}$ ) and is inexpensive and easy. We also aimed to determine the best threshold to distinguish gram $^{-}$sepsis infections from gram $^{+}$sepsis infections.

\section{Methods}

\section{Study subjects}

Between July 2012 and February 2013, study subjects were recruited from infectious inpatient clinics in the Brain Hospital of Hunan Province, China. The study was approved by the ethic committee of our hospital. During this period, a total of 220 subjects (123 males and 97 females) who had data regarding fever [we reviewed the each study patient suffered from high temperature $\left.\left(>38^{\circ} \mathrm{C}\right)\right]$ or infection and were over the age of 18 were recruited. The febrile patients were devided into 3 groups. The patients of two groups were judged by the criteria for systemic inflammatory response syndrome (SIRS, $\mathrm{n}=75)$ and sepsis $(\mathrm{n}=76)$, as described by the 1991 American College of Chest Physicians/Society of Critial Care Medicine Consensus Conference (Bone et al. 1992). The study SIRS sujects met two or more of the following conditions without evidence of a bacterial infection: (1) temperature $>38^{\circ} \mathrm{C}$; (2) heart rate $>90$ beats $/ \mathrm{min}$; (3) respiratory rate $>20$ breaths $/ \mathrm{min}$ or $\mathrm{PaCO}_{2}<32 \mathrm{~mm} \mathrm{Hg}$; (4) WBC count $>12 \times 10^{9} / \mathrm{L},<4 \times 10^{9} / \mathrm{L}$ or $>10 \%$ immature (bands) forms. The sepsis patients were diagnosed by the above-mentioned SIRS criteria and a bacterial infection evidence. The other group of patients with local infect $(n=69)$ only suffered from fever and confirmed infection without SIRS. The definitions for a microbiologically confirmed case of a bacterial infection included isolation of an organism from a culture from the blood $(\mathrm{n}=49)$, urine at $>10^{5} / \mathrm{mL}(\mathrm{n}=18)$, needle aspiration of an abscess or empyema $(n=3)$, sputum sample $(n=68)$, or pharyngeal swab $(n=7)$. Microbiological evaluations confirmed bacterial infections $(n=145)$ in local infection and sepsis patients (Table 1). 
Table 1 Grouping of patients with febrile infection

\begin{tabular}{|c|c|c|}
\hline Group & Bacterial infection & Etiologic agents \\
\hline \multirow[t]{12}{*}{ Local infection (69) } & \multirow[t]{5}{*}{ Gram-positive (gram ${ }^{+}$) infection (27) } & Staphylococcus aureus (6) \\
\hline & & Streptococcus agalactiae (12) \\
\hline & & Streptococcus pyogenes (3) \\
\hline & & Streptococcus constellatus (3) \\
\hline & & Enterococcus faecium (3) \\
\hline & \multirow[t]{7}{*}{ Gram-negative (gram ${ }^{-}$) infection (42) } & Escherichia coli (9) \\
\hline & & Klebsiella pneumoniae (15) \\
\hline & & Enterobacter cloacae (3) \\
\hline & & Enterobacter intermadium (3) \\
\hline & & Pseudomonas aeruginosa (6) \\
\hline & & Stenotrophomonas maltophilia (3) \\
\hline & & Acinetobacter baumannii (3) \\
\hline \multirow[t]{11}{*}{ Sepsis (76) } & \multirow[t]{3}{*}{ Gram-positive (gram ${ }^{+}$) infection (33) } & Staphylococcus aureus (27) \\
\hline & & Staphylococcus epidermidis (3) \\
\hline & & Enterococcus faecium (3) \\
\hline & \multirow[t]{8}{*}{ Gram-negative $\left(\mathrm{gram}^{-}\right)$infection (43) } & Moraxella lacunata (2) \\
\hline & & Acinetobacter baumannii (11) \\
\hline & & Klebsiella pneumoniae (16) \\
\hline & & Burkholderia cepacia (2) \\
\hline & & Escherichia coli (5) \\
\hline & & Pseudomonas aeruginosa (3) \\
\hline & & Stenotrophomonas maltophilia (3) \\
\hline & & Enterobacter aerogenes (1) \\
\hline
\end{tabular}

Subgroups were sorted based on medical and microbiological examination, including isolation of an organism from a culture from the blood, urine, needle aspiration of an abscess or empyema, sputum sample or pharyngeal swab specimens. The number of presented cases was given in parentheses

Samples were selected from three cohorts: 69 cases of local infections (38 males, 31 females, age: $60.9 \pm 21.1$ years), 75 cases of systemic inflammatory response syndrome (41 males, 34 females, age: $56.3 \pm 19.3$ years), and 76 cases of sepsis (44 males, 32 females, age: $55.4 \pm 17.6$ years), with 33 cases of gram $^{+}$infection and 43 cases of gram ${ }^{-}$ infection. All subjects met a above-mentioned standard of sepsis diagnostics based on exclusion from one of the following: (1) recent infectious disease or chronic inflammatory disease; (2) serious hematologic disease; (3) bone marrow transplantation; (4) connective tissue disease or rheumatism; (5) application of inflammation-inhibiting drugs, such as a non-steroidal anti-inflammatory analgesic, steroid medicines, among others; (6) high cholesterol, high blood pressure, or diabetes; or (7) serious liver and/or kidney function insufficiency. A total of 81 serum samples from healthy volunteers were analyzed (47 males, 34 females, age: $52.6 \pm 18.7$ years). Ethics approval for the study protocol and data analysis was obtained from the committee of Brain Hospital of Hunan Province.

\section{Clinical and laboratory examination}

Non-anticoagulant blood samples were taken at $0,1,5$ and 10 days after admission to the hospital. Healthy volunteers provided samples only once. Total cholesterol (TCH), HDLC, triglycerides (TG), apoA 1, apolipoprotein B (apoB), CRP and PCT were measured from blood samples. All measurements, except CRP and PCT, were performed using a 
Siemens ADVIA 2400 automatic biochemical analyzer with original Siemens reagents. CRP was measured using a NingBo MedicalSystem (China). PCT was tested with Roche cobas e411 (Switzerland). At the same time as the blood collection, all of the samples for bacterial culture from suspected infectious subjects were collected. Blood culture (BACTEC 9050, BD) and bacterial incubation (ChangSha ChangJin, China) and determination (Siemens microscan walkaway 40SI, Germany) were used to identify bacteria species.

\section{Statistical analysis}

Descriptive data were reported as the mean-SD. Group differences were tested using analysis of variance (ANOVA). After ANOVA, pairwise group comparisons were performed using an LSD test with homogeneity of variance parameters and a Games-Howell inspection of the heterogeneity of variance parameters. A two-way factorial analysis of variance with repeated measures was used to quantify significant effects during a 10-day hospital admission. A post hoc analysis with a Bonferroni correction for multiple comparisons was also performed. The efficiency of each variable in differentiating between $\operatorname{gram}^{+}(n=33)$ and gram $^{-}(n=43)$ sepsis was evaluated by a receiver operating characteristic (ROC) curve analysis. Based on this analysis, the cutoff values for each variable were determined to simultaneously maximize both the sensitivity and specificity percentages. In addition to sensitivity and specificity, a maximum area under the ROC curve (AUC) was calculated.

Graphpad Prism 5.0 (Graphpad Software, La Jolla, CA, USA) and SPSS 16.0 for windows (SPSS Inc., Chicago, IL, USA) were used for statistical analyses. All testing was two-tailed, and $p$ values below 0.05 indicated statistical significance.

\section{Results}

\section{Serum blood lipid levels and inflammatory state at admission}

In our previous studies, we demonstrated that blood lipids change in patients with gram $^{-}$bacterial infections (Zou et al. 2005). However, we did not consider blood lipids to be a diagnostic for gram $^{-}$or gram ${ }^{+}$sepsis. Here, we investigated whether the patients suffering from sepsis also had changes in blood lipids. We compared the patients with febrile infections with the healthy subjects at admission. We found the TCH was reduced in the patients with a local infection or SIRS $(p<0.001)$. CRP and PCT was increased $(p<0.001)$ and TCH, HDL-C and apoA1 $(p<0.001)$ were decreased in the sepsis patients. However, CRP was increased in the patients with a SIRS $(p<0.001)$, PCT was elevated in all febrile infectious patients $(p<0.001)$ (Table 2). The blood lipid levels changed in the febrile infectious patients in a state of inflammation. Specifically, TCH was inhibited. The above results showed that the blood lipid parameters changed at admission in all patient groups compared to controls, particularly the sepsis patients.

\section{Serum blood lipid levels and inflammatory state at different times after admission}

Because the blood lipid parameters changed at admission in the febrile infectious subjects, we next determined whether the blood lipid parameters had dynamic changes during hospitalization in our study subjects. By monitoring the levels of serum blood lipids at $0,1,5,10$ days after admission, we found that the febrile infectious subjects 
Table 2 Serum blood lipids level and inflammatory state at admission ( $\bar{x} \pm$ SD)

\begin{tabular}{lllllllll}
\hline Group & $\begin{array}{l}\text { Case } \\
(\mathbf{n})\end{array}$ & $\begin{array}{l}\mathrm{TCH} \\
(\mathbf{m m o l} / \mathbf{L})\end{array}$ & $\begin{array}{l}\mathrm{HDL}-\mathrm{C} \\
(\mathbf{m m o l} / \mathbf{L})\end{array}$ & $\begin{array}{l}\mathrm{TG} \\
(\mathbf{m m o l} / \mathbf{L})\end{array}$ & $\begin{array}{l}\text { apoA1 } \\
\mathbf{( g / L})\end{array}$ & $\begin{array}{l}\text { apoB } \\
(\mathbf{g} / \mathbf{L})\end{array}$ & $\begin{array}{l}\text { CRP } \\
(\mathbf{m g} / \mathbf{L})\end{array}$ & $\begin{array}{l}\text { PCT } \\
(\mathbf{n g} / \mathbf{m L})\end{array}$ \\
\hline $\begin{array}{l}\text { Local } \\
\text { infec- } \\
\text { tion }\end{array}$ & 69 & $3.74 \pm 1.22^{* *}$ & $0.91 \pm 0.33$ & $1.22 \pm 0.74$ & $0.95 \pm 0.29$ & $0.84 \pm 0.22$ & $54.3 \pm 96.1^{* *}$ & $0.21 \pm 0.07^{* *}$ \\
SIRS & 75 & $3.67 \pm 1.22^{* *}$ & $0.93 \pm 0.31$ & $1.17 \pm 0.44$ & $0.93 \pm 0.25$ & $0.80 \pm 0.26$ & $62.5 \pm 67.5^{* *}$ & $0.18 \pm 0.10^{* *}$ \\
Sepsis & 76 & $3.41 \pm 1.06^{* *}$ & $0.80 \pm 0.31^{* *}$ & $1.37 \pm 0.84$ & $0.83 \pm 0.31^{* *}$ & $0.83 \pm 0.31$ & $79.5 \pm 94.0^{* *}$ & $0.20 \pm 0.12^{* *}$ \\
Control & 81 & $4.62 \pm 1.05$ & $1.02 \pm 0.22$ & $1.39 \pm 0.82$ & $1.01 \pm 0.16$ & $0.94 \pm 0.23$ & $9.4 \pm 17.4$ & $0.04 \pm 0.02$ \\
\hline
\end{tabular}

Data were expressed as mean values with standard deviation (SD). Patients and control group differences were tested using analysis of variance (ANOVA). After ANOVA, pairwise group comparisons were performed using an LSD test with homogeneity of variance parameters and a Games-Howell inspection of the heterogeneity of variance parameters

${ }^{*} p<0.05 ;{ }^{* *} p<0.01$

had differences in blood lipid levels, sch as TCH $(p=0.003)$, HDL-C $(p<0.001)$ and apoB $(p<0.001)$, and in inflammatory parameter PCT $(p<0.001)$. These levels also significantly changed during the course of the admission in different patients. The HDL-C levels had a tendency to only be reduced in the sepsis group and generally began to drop within $24 \mathrm{~h}$ of admission. The SIRS subjects had elevated TCH and apoB. PCT started to elevate within 5 days after admission in all febrile infectious patients $(p<0.001)$ (Fig. 1). These results illustrate that HDL-C was markedly suppressed in the sepsis patients during hospitalization.

\section{Serum blood lipid levels in the patients with gram ${ }^{-}$sepsis at admission}

In this study, we observed that HDL-C was more greatly inhibited in gram ${ }^{-}$sepsis. Although PCT can indicate patients' inflammatory state, can't distinguish different gram-stained type. From the analysis of the lipids levels of the sepsis patients with different gram-stained bacteria at admission, we found that compared with the patients with gram $^{+}$sepsis, HDL-C $(p<0.001)$ and apoA1 $(p=0.008)$ were significantly reduced in the patients with gram ${ }^{-}$sepsis (Fig. 2; Table 3). The remaining blood lipid parameters were not different between the Gram classifications (Table 3).

\section{Delta HDL-C ratio in detecting gram ${ }^{-}$sepsis}

The HDL-C and apoA1 levels had a tendency to be reduced in the sepsis patients. This decrease was more obvious in the gram $^{-}$sepsis patients. The largest decrease occurred on the sixth day after admission. Interestingly, in contrast to gram $^{+}$sepsis, gram $^{-}$sepsis induced a potent downregulation of HDL-C on the sixth day. We determined how much $\mathrm{HDL}-\mathrm{C}$ had changed in the gram ${ }^{-}$sepsis patients by calculating the ratio of the HDL-C decrease $\left[\left(\frac{H D L-C_{1 d}-H D L-C_{0 d}}{H D L-C_{0 d}}\right) \times 100 \%\right]$ and conducting a ROC curve analysis on the gram $^{-}$sepsis patients within $24 \mathrm{~h}$ of admission.

The ROC curve analysis confirmed that the delta ratio of HDL-C was different between the gram $^{-}$sepsis and gram ${ }^{+}$sepsis patients and had a $70.5 \%$ specificity and $76.5 \%$ sensitivity. The area under the curve was $0.744(\mathrm{SE}=0.057, p<0.001)$. The highest observed value $-21.1 \%$ (Fig. 3; Table 4). 

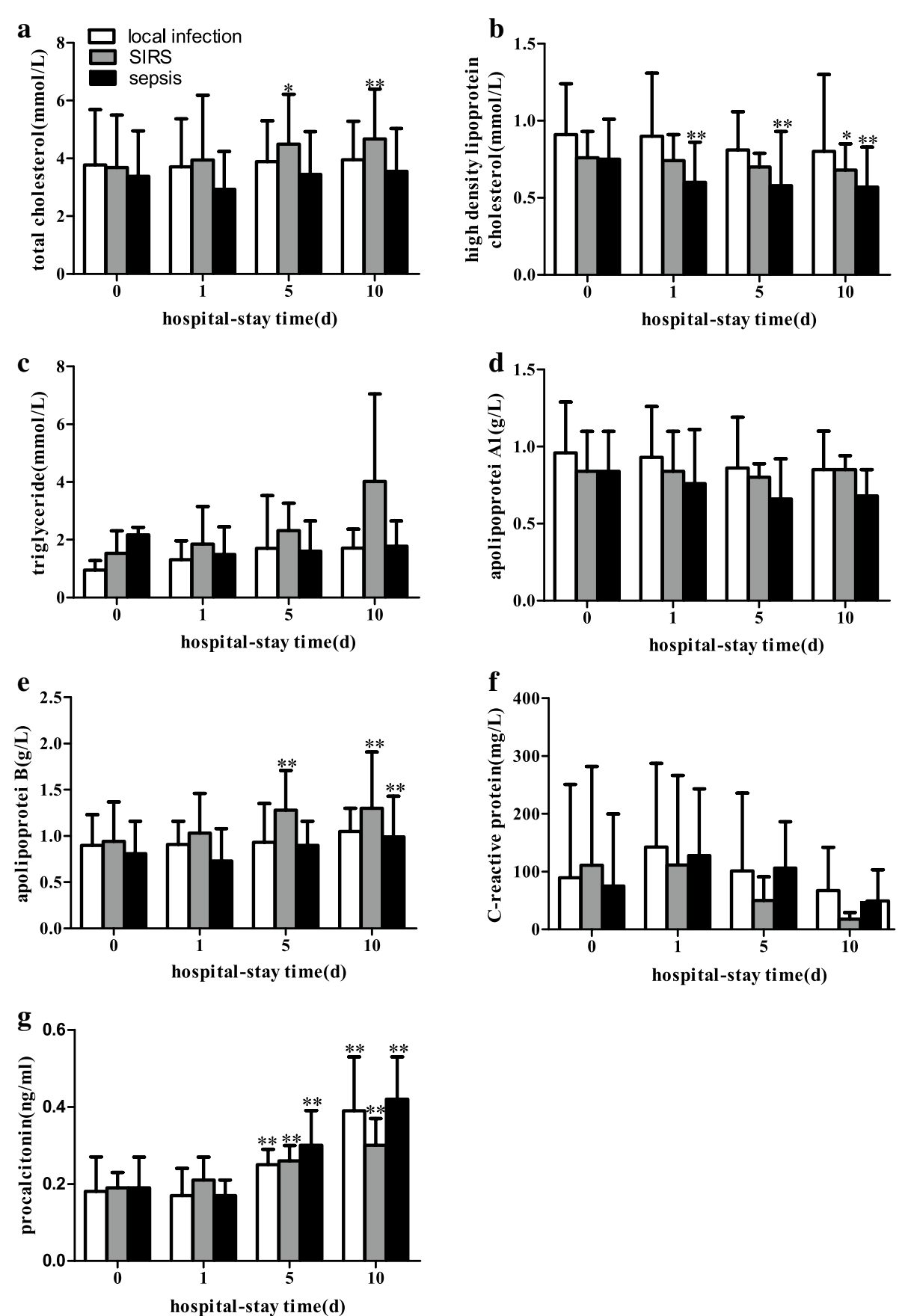

Fig. 1 Serum lipid levels and inflammatory state at different times after admission. a Total cholesterol; b high-density lipoprotein cholesterol; $\mathbf{c}$ triglycerides; $\mathbf{d}$ apolipoprotein $\mathrm{A} 1$; e apolipoprotein $\mathrm{B} ; \mathbf{f} \mathrm{C}$-reactive protein; $\mathbf{g}$ procalcitonin. The box chart statistics (mean value and SD) of the data are also shown. Two-way factorial analysis of variance with repeated measures was used to quantify the main effects during the 10-day hospital admission. The post hoc analysis was performed using a Bonferroni correction for multiple comparisons 

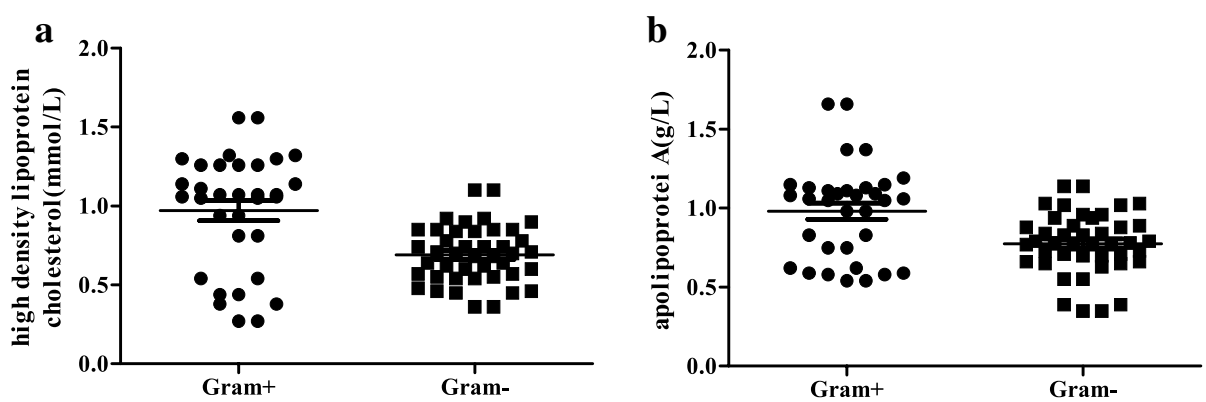

Fig. 2 Influence of different Gram stain bacterial sepsis on the blood lipid levels at admission. a High-density lipoprotein cholesterol. b Apolipoprotein A1. The box chart statistics (mean value and SEM). The difference between the $\mathrm{Gram}^{+}$and $\mathrm{Gram}^{-}$sepsis groups were tested using an independent-samples T test

Table 3 Serum blood lipids levels and inflammatory state in gram $^{-}$sepsis at admission $(\bar{x} \pm$ SD)

\begin{tabular}{lllllllll}
\hline Group & $\begin{array}{l}\text { Case } \\
(\mathbf{n})\end{array}$ & $\begin{array}{l}\text { TCH } \\
(\mathbf{m m o l} / \mathbf{L})\end{array}$ & $\begin{array}{l}\text { HDL-C } \\
(\mathbf{m m o l} / \mathbf{L})\end{array}$ & $\begin{array}{l}\text { TG } \\
(\mathbf{m m o l} / \mathbf{L})\end{array}$ & $\begin{array}{l}\text { apoA1 } \\
(\mathbf{g} / \mathbf{L})\end{array}$ & $\begin{array}{l}\text { ApoB } \\
(\mathbf{g} / \mathbf{L})\end{array}$ & $\begin{array}{l}\text { CRP } \\
(\mathbf{m g} / \mathbf{L})\end{array}$ & $\begin{array}{l}\text { PCT } \\
(\mathbf{n g} / \mathbf{m L})\end{array}$ \\
\hline $\begin{array}{c}\mathrm{Gram}^{+} \\
\text {sepsis }\end{array}$ & 33 & $3.69 \pm 0.99$ & $0.97 \pm 0.37$ & $1.50 \pm 1.59$ & $0.98 \pm 0.30$ & $0.87 \pm 0.35$ & $69.9 \pm 93.6$ & $0.17 \pm 0.10$ \\
$\begin{array}{c}\mathrm{Grm}^{-} \\
\text {sepsis }\end{array}$ & 43 & $3.14 \pm 0.95$ & $0.69 \pm 0.18^{* *}$ & $1.44 \pm 0.93$ & $0.78 \pm 0.19^{* *}$ & $0.78 \pm 0.24$ & $85.9 \pm 95.2$ & $0.24 \pm 0.13$ \\
\hline
\end{tabular}

Data were expressed as mean values with standard deviation (SD). $\mathrm{Gram}^{+}(n=33)$ and gram ${ }^{-}(n=43)$ sepsis group differences were tested using independent-samples $T$ test

${ }^{*} p<0.05 ;{ }^{* *} p<0.01$

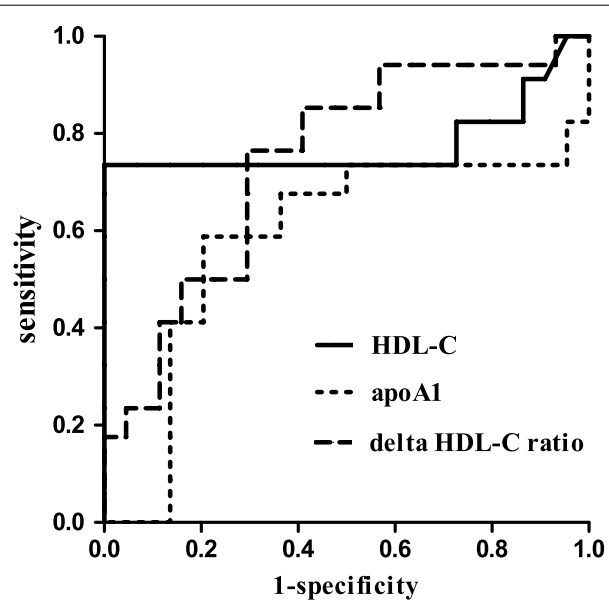

Fig. 3 Receiver operating characteristic (ROC) curves comparing the serum HDL-C and apoA1 levels and the $24 \mathrm{~h}$ change ratio of HDL-C for distinguishing between gram ${ }^{+}$sepsis $(n=33)$ and gram ${ }^{-}$sepsis $(n=43)$. The statistics of the ROC curve analysis and the significance of pairwise comparisons between the areas under the ROC curve (AUC) are also shown

\section{Discussion}

Because severe sepsis and septic shock can be fatal within hours, it is customary to start empirical broad-spectrum antimicrobial therapy in all patients hospitalized with the suspicion of systemic inflammatory response syndrome (Bochud et al. 2004), However, the 
Table 4 Statistic of ROC curve analysis

\begin{tabular}{llll}
\hline & HDL-C & apoA1 & Delta HDL-C ratio \\
\hline Cutoff point & $<0.86$ & $<0.86$ & $<-21.1 \%$ \\
AUC (SE) & $0.777(0.065)$ & $0.586(0.072)$ & $0.744(0.057)$ \\
Sensitivity (\%) & 73.5 & 58.8 & 76.5 \\
Specificity (\%) & 100.0 & 79.5 & 70.5 \\
PPV (\%) & 100.0 & 67.9 & 65.8 \\
NPV (\%) & 82.7 & 70.8 & 78.9 \\
PLR & $\infty$ & 2.87 & 2.59 \\
NLR & 0.27 & 0.52 & 0.42 \\
$p$ value & $<0.001$ & 0.197 & $<0.001$ \\
\hline
\end{tabular}

Data were expressed as the statistics of ROC curve analysis and the significance of pairwise comparisons between gram ${ }^{+}$ sepsis $(n=33)$ and gram ${ }^{-}$sepsis $(n=43)$

overuse of broad-spectrum antimicrobials without bacterial identification has contributed to the emergence of drug-resistant strains of bacteria, especially in ICU patients. For example, first identifying the causative pathogen of an infection and then using an appropriate narrow-spectrum antibiotic against gram $^{+}$or gram $^{-}$bacteria should be a standard procedure to prevent antimicrobial overuse and resistance. However, microbial cultures are time consuming. Therefore, determining a new quick method to determine the type of infecting pathogen is essential.

We have proven in our previous studies that HDL-C and apoA 1 are significantly decreased in patients with a gram ${ }^{-}$infection. Continuously low levels of HDL-C and apoA1 hint at an uncontrolled infection (Zou et al. 2006). In addition, patients with a gram $^{+}$infection have none of the above changes. Similarly, in this study, we found that blood lipids changed in the patients with all types of infections. TCH, HDL-C and apoA1 were significantly lower in the sepsis subjects. HDL-C appeared to gradually trend downward from admission after 1 day and was positively related to the severity of disease. As Kumaraswamy et al. (2012) demonstrated, HDL-C was greatly reduced in sepsis and SIRS, with the lowest HDL-C levels in sepsis shock. The degree of HDL-C reduction was associated with the severity of disease. We found in the SIRS group total cholesterol levels increased after day 5 and HDL-C did not change until day 10, this was not consistent with the finding of Kumaraswamy SB, which TCH and HDL-C were decrease. We observed different time period. The study test of Kumaraswamy SB was within $24 \mathrm{~h}$. Moreover, there may be the treatment of the SIRS patients was effective during 10 day, thereby acute-phase proteins HDL-C did not fall, and the patients had good prognosis, so the every sick can survive.

In recent years, a large number of animal studies have found that HDL can combine with and neutralize the lipopolysaccharide (LPS) of gram-negative bacteria (Khovidhunkit et al. 2004). Lipoproteins bind with bacterial cell walls and cholesterol-loaded macrophages fight against LPS during bacterial infections. HDL selectively inhibits IFN I gene activity, thus playing a role inregulating innate immune function (Suzuki et al. 2010). Studies have shown that in vitro, pure LPS combines with $60 \%$ HDL (Levels et al. 2001), and there are similar results with lipoteichoic acid (LTA) (Levels et al. 2003). This shows that HDL changes are related to gram ${ }^{-}$bacterial infections. 
In this study, we reported that decreased HDL-C levels correlate with the likelihood of gram ${ }^{-}$bacterial infections, especially gram ${ }^{-}$sepsis. HDL contains both proteins and cholesterol, but the cholesterol content is relatively stable and less influenced by the diet and nutritional status. The degree of variation of HDL-C was $0.7 \%$. For the first time, we were able to demonstrate a novel parameter for gram ${ }^{-}$sepsis, the $24 \mathrm{~h}$ delta HDL-C ratio. Although the HDL-C ratio can't be used until after $24 \mathrm{~h}$ after admission, which seemingly makes the time-difference to the gram stain method the same. The gram stain method can be implemented after cultivating bacteria, which may require 24 to $48 \mathrm{~h}$, such as blood culture, in particular the slowly grown bacteria. The advantages of the delta HDL-C ratio over pathogen culture include that it is based on a routine measurement of HDL-C and is convenient, economical and assured quality. Trial specimen is more easy to get, collected conveniently. It takes less than an hour to receive HDL-C results, and the test can be performed quickly at any time without advanced technology.

In an in vitro experiment, HDL combined with bacterial LPS via an LPS binding protein, thereby reducing TLR4 expression and signal transduction (Van Linthout et al. 2011), down-regulating the NF-kappa B pathway and alleviating inflammation and mortality induced by LPS. Clinical studies by Van Leeuwen et al. (2003) have shown that lipoprotein levels are significantly decreased in ICU sepsis patients and are positively correlated with mortality. Gruber et al. (2009) demonstrated a similar phenomenon in lower respiratory tract infections. The CRP values decreased to $1.01 \mathrm{mg} / \mathrm{dl}$ and HDL-C to $0.18 \mathrm{mmol} / \mathrm{L}$ in the disease. The level of $0.82 \mathrm{mmol} / \mathrm{L}$ HDL-C was indicative of respiratory infections. The present study suggests that the blood lipid levels changed in the febrile infectious patients at admission. TCH, HDL-C and apoA1 decreased, particularly in sepsis patients (Table 2). Therefore, we surveyed the blood lipid parameter changes throughout hospitalization. The results demonstrated that HDL-C levels had a sustained decline starting at $24 \mathrm{~h}$ after admission in the sepsis patients, which was different from the SIRS and local infections (Fig. 1).

Sepsis developed quickly and led to a high mortality rate. Sepsis has often been treated with broad spectrum antibiotics, which has led to an emergence of resistant strains. Thus, first distinguishing between gram $^{+}$or gram $^{-}$sepsis is helpful in choosing the appropriate antibiotics for effective treatment. Thus, an accurate method is needed to effectively and quickly identify the types of pathogenic bacteria involved in sepsis. Recently, PCT and CRP can predict and identify patients for suspected sepsis in an emergency department (Tsalik et al. 2012). Futhermore, studies have reported signifi cantly higher levels in infections caused by gram-negative organisms than gram-positive or fungal infections (Brodska et al. 2013). However, another study showed that the PCT levels are higher in patients with $S$. aureus infection than in those with coagulasenegative staphylococci (Shomali et al. 2013). These indicated the PCT level is affected by a variety of infectious Bacterial species, so it is difficult to distinguish between gram staining sepsis, and we found PCT can't distinguish between gram $^{-}$and gram ${ }^{+}$sepsis (Table 3).

Berbee et al. (2005) found that in animals, HDL can combine with the cell walls of gram $^{-}$LPS and weaken the body's biological reaction. They also found that HDL can protect rodents from death caused by sepsis. In this study, we tested the lipid levels in gram ${ }^{+}$ or gram $^{-}$sepsis patients and found that HDL-C was reduced within 1 day after admission 
in the gram $^{-}$sepsis patients (Table 3; Fig. 2). According to the ROC curve analysis, the delta ratio of HDL-C displayed a $70.5 \%$ specificity and $76.5 \%$ sensitivity for the detection of gram $^{-}$sepsis in the febrile patients with bacterial infections. The critical value was $-21.1 \%$ (Table 4). Studies have shown that gram stain of the knee aspirates was $45 \%$ sensitivity and $100 \%$ specificity in acute septic arthritis (Faraj et al. 2002), the sensitivity and specificity of Gram stain of liver abscesses aspirates were 52 and $94 \%$ for gramnegative bacilli (GNB) and the sensitivity of the blood cultures for any GNB present were 39 \% in liver abscesses patients (Chemaly et al. 2003). These show gram stain have lower sensitivity. In our retrospective study, most of objects hadn't results of Gram stain before culture. So unfortunately we can't compare the delta ratio of HDL-C with Gram stain before culture in gram ${ }^{-}$sepsis. Interestingly, HDL-C had an $100 \%$ specificity and $73.5 \%$ sensitivity (Fig. 3), which was superior to the delta HDL-C ratio. Yildiz et al. (2009) also showed that in late onset neonatal sepsis, the HDL-C levels were significantly lower, with a $96.2 \%$ specificity and $44.4 \%$ sensitivity. However, there are other factors that influence the HDL-C levels, such as the nutritional status, liver status, lipid metabolism, and so on. Moreover, we studied retrospective, typical cases. Finally, we considered the change rate of HDL-C in gram ${ }^{-}$bacterial sepsis patients. The 24 -h time point sample was determined to be the best for observing an initial change. ApoA1 was not different between the gram ${ }^{+}$ and gram $^{-}$sepsis patients after $24 \mathrm{~h}$. In summary, the delta decrease ratio was the most appropriate measurement to detect gram- sepsis compared with the individual variation in the serum HDL-C and apoA1 levels. Unfortunately, there still means that $23.5 \%$ of the patients tested gram $^{+}$sepsis are actually gram ${ }^{-}$sepsis and $29.5 \%$ that are tested gram $^{-}$ sepsis are actually gram ${ }^{+}$sepsis. A research has shown that patients with septic shock associated with GNB infection can decrease the survival rate of patients 3.5-fold, survival fraction changes of 3.9-fold with inappropriate initial empiric therapy (Kumar et al. 2009). So it was not enough only with the HDL-C ratio distinguish between gram $^{-}$and gram $^{+}$ sepsis. If a joint detection of the delta HDL-C ratio and HDL-C were used, the specificity would increase to $100 \%$, which will reduced the mortality of sepsis patients.

Patients with gram ${ }^{-}$sepsis account for approximately $60 \%$ (24/40)of patients with ICU sepsis. gram $^{-}$sepsis also has a long disease course. About $70 \%$ of patients are still in a severe state five days after admission, which is noted by HDL-C being maintained at a low level. In the prognosis of our study subjects, two cases sepsis patients in vegetative state, sepsis prognosis of four cases were death after 10 days, while all patients survived in other groups. One departed gram ${ }^{+}$sepsis patient, the $24 \mathrm{~h}$ HDL-C reduced $13.08 \%$, other three non-survivors were gram ${ }^{-}$sepsis, the $24 \mathrm{~h} \mathrm{HDL-C} \mathrm{decreased} 25,31.43,50.59 \%$, respectively. HDL-C may have a protective effect against sepsis. In previous hospital studies, every HDL increase of $1 \mathrm{mg} / \mathrm{mL}$ reduced the occurrence rate of severe sepsis by $3 \%$ (Grion et al. 2010). HDL levels above a critical value can indicate gram ${ }^{-}$sepsis. These parameters can assist in determining the appropriate antibiotics to use for sepsis in a timely and effective manner, thus reducing antibiotic overuse and decreasing mortality.

No single method is dependable enough to confirm a fast etiologic diagnosis of sepsis. Instead, a final diagnosis should be determined by a doctor based on a sepsis diagnosis guide. The delta HDL-C ratio test could provide additional laboratory proof to rapidly and conveniently choose an appropriate antibiotic treatment in suspected sepsis. 


\section{Conclusions}

Sepsis patients had lower HDL-C. The 24-h HDL-C change ratio can be used as a novel sepsis diagnostic marker and as a convenient test to determine the type of bacteria involved in an infection. This information can be used to choose appropriate antibiotics and to quickly rescue patients, thereby reducing mortality.

\section{Abbreviations}

HDL-C: high-density lipoprotein cholesterol; apoA 1: apolipoprotein A 1; TCH: total cholesterol; TG: triglycerides; apoB: apolipoprotein B; CRP: C-reactive protein; PCT: procalcitonin; LPS: lipopolysaccharide; Gram': gram-negative bacteria; $\mathrm{Gram}^{+}$: gram-positive bacteria; SIRS: systemic inflammatory response syndrome; GNB: gram-negative bacilli.

\section{Authors' contributions}

GZ contributed to the conception of this work and prepared the manuscrip; JH and GX performed the experiments; FX analyzed the data; BR and WZ helped perform the analysis with constructive discussions. All authors read and approved final manuscript.

\section{Author details}

${ }^{1}$ Department of Clinical Laboratory, Brain Hospital of Hunan Province, Furong Middle Road 427, Changsha 410007, Hunan, People's Republic of China. ${ }^{2}$ Department of Medical Laboratory, Hunan University of Traditional Chinese Medicine, Changsha, People's Republic of China. ${ }^{3}$ Department of Laboratory Medcine, Xiangya Medical School, Central South University, Changsha, People's Republic of China.

\section{Acknowledgements}

This work was supported by the Health and Family Planning Commission of Hunan Province plan projects (B2014-103) and the Hunan province science and technology plan projects (Grant No. 2012FJ6073).

\section{Competing interests}

The authors declare that they have no competing interests.

Received: 19 December 2015 Accepted: 23 June 2016

Published online: 11 July 2016

\section{References}

Berbee JF, Havekes LM, Rensen PC (2005) Apolipoproteins modulate the inflammatory response to lipopolysaccharide. J Endotoxin Res 11(2):97-103

Bochud PY, Bonten M, Marchetti O, Calandra T (2004) Antimicrobial therapy for patients with severe sepsis and septic shock: an evidence-based review. Crit Care Med 32(11 Suppl):S495-S512

Bone RC, Balk RA, Cerra FB, Dellinger RP, Fein AM, Knaus WA et al (1992) Definitions for sepsis and organ failure and guidelines for use of innovative therapies in sepsis: ACCP/SCCM Consensus Conference Committee; American College of Chest Physicians/Society of Critical Care Medicine. Chest 101(6):1644-1655

Brodska H, Malickova K, Adamkova V, Benakova H, Stastna MM, Zima T (2013) Signifi cantly higher procalcitonin levels could differentiate Gram-negative sepsis from Gram-positive and fungal sepsis. Clin Exp Med. 13(3):165-170

Chemaly RF, Hall GS, Keys TF, Procop GW (2003) Microbiology of liver abscesses and the predictive value of abscess gram stain and associated blood cultures. Diagn Microbiol Infect Dis 46(4):245-248

Faraj AA, Omonbude OD, Godwin P (2002) Gram staining in the diagnosis of acute septic arthritis. Acta Orthop Belg 68(4):388-391

Feingold KR, Funk JL, Moser AH, Shigenaga JK, Rapp JH, Grunfeld C (1995) Role for circulating lipoproteins in protection from endotoxin toxicity. Infect Immun 63(5):2041-2046

Grion CM, Cardoso LT, Perazolo TF, Garcia AS, Barbosa DS, Morimoto HK et al (2010) Lipoproteins and CETP levels as risk factors for severe sepsis in hospitalized patients. Eur J Clin Invest 40(4):330-338

Gruber M, Christ-Crain M, Stolz D, Keller U, Muller C, Bingisser R et al (2009) Prognostic impact of plasma lipids in patients with lower respiratory tract infections -an observational study. SWiSS Med Wkly 139(11-12):166-172

Hospodsky D, Yamamoto N, Peccia J (2010) accuracy, precision, and method detection limits of quantitative pcr for airbornebacteria and fungi. Appl Environ Microbiol 76(21):7004-7012

Khovidhunkit W, Kim MS, Memon RA, Shigenaga JK, Moser AH, Feingold KR et al (2004) Effects of infection and inflammation on lipid and lipoprotein metabolism: mechanisms and consequences to the host. J Lipid Res 45(7):1169-1196

Kumar A, Ellis P, Arabi Y, Roberts D, Light B, Parrillo JE et al (2009) Initiation of inappropriate antimicrobial therapy results in a five-fold reduction of survival in human septic shock. Chest 136(5):1237-1248

Kumaraswamy SB, Linder A, Åkesson P, Dahlbäck B (2012) Decreased plasma concentrations of apolipoprotein M in sepsis and systemic inflamatory response syndromes. Crit Care 16(2):R60

Levels JH, Abraham PR, van Barreveld EP, Meijers JC, van Deventer SJ (2001) Distribution and kinetics of lipoproteinbound endotoxin. Infect Immun 69(5):2821-2828

Levels JH, Abraham PR, van Barreveld EP, Meijers JC, van Deventer SJ (2003) Distribution and kinetics of lipoproteinbound lipoteichoic acid. Infect Immun 71(6):3280-3284 
Shomali W, Hachem R, Chaftari AM, Bahu R, Helou GE, Jiang Y et al (2013) Can procalcitonin differentiate Staphylococcus aureus from coagulase-negative staphylococci in clustered gram-positive bacteremia? Diagn Microbiol Infect Dis 76(2):158-161

Suzuki M, Pritchard DK, Becker L, Hoofnagle AN, Taimura N, Bammler TK et al (2010) High-density lipoprotein suppresses the type I interferon response, a family of potent antiviral immunoregulators, in macrophages challenged with lipopolysaccharide. Circulation 122(19):1919-1927

Thaveeratitham P, Plengpanich W, Naen-Udorn W, Patumraj S, Khovidhunkit W (2007) Effects of human apolipoprotein Aon endotoxin-induced leukocyte adhesion on endothelial cells in vivo and on the growth of Escherichia coli in vitro. J Endotoxin Res 13(1):58-64

Tsalik EL, Jaggers LB, Glickman SW, Langley RJ, van Velkinburgh J, Park LP et al (2012) Discriminative value of infl ammatory biomarkers for suspected sepsis. J Emerg Med 43(1):97-106

Van Leeuwen HJ, Heezius EC, Dallinga GM, van Strijp JA, Verhoef J, van Kessel KP (2003) Lipoprotein metabolish in patients with severe sepsis. Crit Care Med 31(5):1359-1366

Van Linthout S, Spillmann F, Graiani G, Miteva K, Peng J, Van Craeyveld E et al (2011) Down-regulation of endothelial TLR4 signalling after apoA-I gene transfer contributes to improved survival in an experimental model of lipopolysaccharide-induced inflammation. J Mol Med 89(2):151-160

Yildiz B, Ucar B, Aksit A, Aydogdu SD, Colak O, Colak E (2009) Diagnostic values of lipid and lipoprotein levels in late onset neonatal sepsis. Scand J Infec Dis 41(4):263-267

Zou GY, Hu LP, Ren BQ (2005) Clinical significance of the mensurations of plasma lipids in Gram-negative bacteremia patients. J Mod Lab Med 20(6):69-70

Zou GY, Hu LP, Ren BQ (2006) Clinical significance of the mensurations of plasma high density lipoprotein cholesterol and apolipoprotein A1 in Gram- negative bacillus infection patients. Int J Lab Med 27(3):280-281

\section{Submit your manuscript to a SpringerOpen ${ }^{\odot}$ journal and benefit from:}

- Convenient online submission

\section{Rigorous peer review}

- Immediate publication on acceptance

- Open access: articles freely available online

- High visibility within the field

- Retaining the copyright to your article

Submit your next manuscript at $\boldsymbol{\nabla}$ springeropen.com 